OPEN ACCESS

Edited by:

Li-Tung Huang,

Kaohsiung Chang Gung Memorial

Hospital, Taiwan

Reviewed by:

Stefania Gallucci,

Temple University, United States

Roberto Caricchio,

Temple University, United States

Mei-Hsin Hsu,

Kaohsiung Chang Gung Memorial

Hospital, Taiwan

Jun-Jun Yeh,

Ditmanson Medical Foundation

Chia-Yi Christian Hospital, Taiwan

${ }^{*}$ Correspondence:

Renin Chang

rhapsody1881@gmail.com

Hsin-Hua Chen

shc5555@hotmail.com

James Cheng-Chung Wei

wei3228@gmail.com

${ }^{+}$These authors have contributed equally to this work and share first authorship

Specialty section:

This article was submitted to

Autoimmune and

Autoinflammatory Disorders,

a section of the journal

Frontiers in Immunology

Received: 16 June 2021 Accepted: 28 October 2021 Published: 23 November 2021

Citation:

Tu T-Y, Yeh $C-Y$, Hung $Y-M$,

Chang $\mathrm{R}$, Chen $\mathrm{H}-\mathrm{H}$ and

Wei JC-C (2021) Association between a History of Nontyphoidal Salmonella and the Risk of Systemic Lupus Erythematosus: A Population-

Based, Case-Control Study.

Front. Immunol. 12:725996. doi: 10.3389/fimmu.2021.725996

\section{Association Between a History of Nontyphoidal Salmonella and the Risk of Systemic Lupus Erythematosus: A Population-Based,
Case-Control Study}

Ting-Yu Tu ${ }^{1+}$, Chiu-Yu Yeh ${ }^{2 \dagger}$, Yao-Min Hung ${ }^{3,4,5,6}$, Renin Chang ${ }^{7 *}$, Hsin-Hua Chen ${ }^{6,8,9,10,11 *}$ and James Cheng-Chung Wei ${ }^{4,12,13^{*}}$

\begin{abstract}
${ }^{1}$ Department of Orthopedics, Kaohsiung Veterans General Hospital, Kaohsiung, Taiwan, ${ }^{2}$ Department of Medicine, School of Medicine, Kaohsiung Medical University, Kaohsiung, Taiwan, ${ }^{3}$ College of Health and Nursing, Meiho University, Pingtung, Taiwan, ${ }^{4}$ Institute of Medicine, Chung Shan Medical University, Taichung, Taiwan, ${ }^{5}$ Department of Internal Medicine, Kaohsiung Municipal United Hospital, Kaohsiung, Taiwan, ${ }^{6}$ School of Medicine, National Yang Ming University, Taipei, Taiwan, ${ }^{7}$ Department of Emergency Medicine, Kaohsiung Veterans General Hospital, Kaohsiung, Taiwan, ${ }^{8}$ Division of Allergy, Immunology and Rheumatology, Division of General Internal Medicine, Department of Internal Medicine, Taichung Veterans General Hospital, Taichung, Taiwan, 9 Institute of Biomedical Science and Rong Hsing Research Centre for Translational Medicine, Chung Hsing University, Taichung, Taiwan, ${ }^{10}$ Department of Industrial Engineering and Enterprise Information, Tunghai University, Taichung, Taiwan, ${ }^{11}$ Institute of Public Health and Community Medicine Research Center, National Yang-Ming University, Taipei, Taiwan, ${ }^{12}$ Graduate Institute of Integrated Medicine, China Medical University, Taichung, Taiwan, ${ }^{13}$ Division of Allergy, and Institute of Medicine, Chung Shan, Medical University, Immunology and Rheumatology, Taichung, Taiwan
\end{abstract}

Objective: We investigated the correlation between nontyphoidal Salmonella (NTS) infection and systemic lupus erythematosus (SLE) risk.

Methods: This case-control study comprised 6,517 patients with newly diagnosed SLE between 2006 and 2013. Patients without SLE were randomly selected as the control group and were matched at a case-control ratio of 1:20 by age, sex, and index year. All study individuals were traced from the index date back to their NTS exposure, other relevant covariates, or to the beginning of year 2000. Conditional logistic regression analysis was used to analyze the risk of SLE with adjusted odds ratios (aORs) and 95\% confidence intervals (Cls) between the NTS and control groups.

Results: The mean age was 37.8 years in the case and control groups. Females accounted for $85.5 \%$. The aOR of having NTS infection were significantly increased in SLE relative to controls (aOR, 9.20; 95\% Cl, 4.51-18.78) in 1:20 sex-age matching analysis and (aOR, 7.47; 95\% $\mathrm{Cl}=2.08-26.82)$ in propensity score matching analysis. Subgroup analysis indicated that the SLE risk was high among those who dwelled in rural areas; had rheumatoid arthritis, multiple sclerosis, or Sjogren's syndrome; and developed intensive and severe NTS infection during admission. 
Conclusions: Exposure to NTS infection is associated with the development of subsequent SLE in Taiwanese individuals. Severe NTS infection and other autoimmune diseases such as rheumatoid arthritis, multiple sclerosis, or Sjogren's syndrome also contributed to the risk of developing SLE.

Keywords: nontyphoidal Salmonella, systemic lupus erythematosus, NHIRD, case-control study, immunology, NTS, SLE, epidemiology

\section{HIGHLIGHTS}

- This population-based, case-control study suggested a significant association between a history of nontyphoidal Salmonella (NTS) infection and systemic lupus erythematosus (SLE).

- NTS may be a potential trigger for SLE via signaling of proinflammatory cytokines produced by innate and adaptive immune responses.

- A massive data analysis containing approximately $99 \%$ of Taiwan's population of 23 million people

\section{INTRODUCTION}

Systemic lupus erythematosus (SLE), whose global prevalence and incidence respectively range from $9-241$ and $0.3-23.2$ per $1,00,000$ person-years (1), is among the top 20 leading causes of death in women aged 5-64 years (2). The multifactorial etiology of SLE, including genetic factors, immune dysfunction-related factors, environmental and hormonal factors, can be deemed as the result of exposome-epigenome-genome interactions (3). Genetic susceptibility alone, however, is insufficient to trigger the onset of SLE, with the evidence of only $24 \%$ concordance rates among monozygotic twins with SLE manifestation (4). This indicates that besides genetic factors, the environment also plays a crucial role in the pathophysiology of SLE (5). In addition, heritability, shared (familial) environmental-, and nonshared environmental factors were reported to account for SLE susceptibility in $43.9 \%, 25.8 \%$, and 30.3\% Taiwanese patients, respectively (6).

The major environmental factors influencing SLE development include exposure to ultraviolet radiation, particulate air pollution, trace elements, alcohol use, and infections (7). A correlation between persistent infections and the development of autoimmune diseases has been reported. Infections by viruses, especially Epstein-Barr virus, parvovirus B19, cytomegalovirus, varicella-zoster virus (8), and retrovirus, might play a pivotal pathogenetic role in SLE and are highly associated with SLE (9). Likewise, bacterial infections are also linked to SLE. Gut commensals, for instance, were reported to contribute to the immune pathogenesis of lupus nephritis (10). Additionally, a common anti-DNA idiotype (16/6Id) carried by antibodies against Mycobacterium tuberculosis accounts for the antinuclear autoantibody positivity in tuberculosis patients (11), which may lead to autoimmune phenomena (12).
The association between salmonellae infections and the development of autoimmune diseases (13-16) has been reported recently. Salmonella enterica serovar Typhimurium and Enteritidis seem to act more aggressively than other bacterial infections in SLE patients. Other than causing localized gastroenteritis, salmonella infection in SLE patients can cause bacteremia and complications with high mortality rates $(17,18)$.

Nontyphoidal Salmonellae (NTS) is a major cause of diarrhea and foodborne gastroenteritis worldwide, and the risk of invasive gastroenteritis is six-fold higher than that of other bacterial infections (19). The global burden of NTS gastroenteritis was estimated to be 94 million cases and 1,55,000 deaths yearly worldwide, with an especially high incidence of 4 cases per 100 persons and over 88,000 associated deaths in 2006 in east Asia (20).

Salmonella infection due to defective Toll-like receptor (TLR)/myeloid differentiation primary response 88 signaling results in disruption of the cellular plasma membrane in B cells, highlighting the importance of correlations between genotype and environmental factors (21).

We hypothesized that a past NTS infection increases the risk of subsequent SLE. As the epidemiological relationship between NTS and SLE has not been clarified, we conducted this original nationwide case-control study to address this important issue.

\section{METHODS}

\section{Data Source}

This study was conducted using data from Taiwan's National Health Insurance Research Database (NHIRD), which consists of original claim data and registration files for almost $99 \%$ of Taiwan's population. Registry for Catastrophic Illness Patients (RFCIP) belongs to a subset of NHIRD that lists diseases requiring long-term care. Patients in RFCIP are exempted from medical expenses as to protect vulnerable beneficiaries. Therefore, the reliability and accuracy of SLE diagnosis received by the enrolled patients were assured. We also employed another subset of NHIRD, the Longitudinal Health Insurance Research Database (LHIRD), in this study. The LHIRD comprises all the original claims data of 1,000,000 people randomly sampled from the year 1997 to 2013 registry of the NHIRD. The positive predictive value of NHIRD claims data can be up to $84.6 \%$ with a strict definition of study group selection. In Taiwan, hospital-licensed medical records technicians validated the coding before claiming the reimbursements, and the National 
Health Insurance Administration authority verified the audit. The Institutional Review Board of Taichung Veterans General Hospital (TCVGH CE14149B-1) approved this study.

\section{Study Population}

As shown in Figure 1, we identified patients who had been diagnosed with SLE by the International Classification of Diseases, Ninth Revision (ICD-9) codes (710.0) in the RFCIP during the period 1997-2013 $(n=16,487)$. The index date was defined as the first date of SLE diagnosis during 2006-2013. Individuals diagnosed with SLE before 2006 were excluded. The control group was randomly selected from LHIRD during 20062013, which contained outpatients and inpatients data and was matched at a ratio of 1:20 by age, sex, and index year. Totally, 6,517 patients were included in the SLE group, and 130,340 nonSLE individuals served as the control group. We further performed propensity score matching (PSM) at a $1: 2$ ratio to minimize the potential confounding effects of sex, age, and selected comorbidities on the incidence of SLE.

\section{Exposure and Potential Confounders}

The exposure of interest in this study was NTS (ICD-9-CM code 003.xx). The exposure/covariate assessment period was set from the year 2000 to the index date. Quantiles were often used in the preliminary assessment of exposure-outcome relationships, and we applied quartiles for cut-offs. To eliminate potential confounding bias, we adjusted for demographic variables and relevant comorbidities whose relative risks are significant to SLE according to previous studies $(6,22)$. These comorbidities, selected based on at least three outpatient visits or one admission, included psoriasis (ICD-9-CM code 696.1), psoriatic arthropathy (696.0), inflammatory bowel disease (555-556), Crohn's disease (555), ulcerative colitis (556), ankylosing spondylitis (720.0), Sjogren's syndrome (710.2), rheumatoid arthritis (714.0), tonsillitis (474.0, 463), psychosocial status (290-319), chronic obstructive pulmonary disease (COPD; 491-492, 493-496), alcohol-related illness (303, 305), chronic liver disease (571.4), cancer (140-239), and multiple sclerosis (340). High-risk drugs (23) for SLE with an administration duration of more than 28 days, such as procainamide (ATC code C01BA02), hydralazine (C02DB02), and isoniazid (J04AC01), were included. Gingival and periodontal diseases (ICD-9-CM code 253) were diagnosed by dentists and included based on the use of one of the following procedures/medications: (1) AHFS code: antibacterials (081200), aminoglycosides (081202), antifungal antibiotics (081204), cephalosporins (081206), miscellaneous beta-lactam antibiotics (081207), chloramphenicol (081208), macrolides (081212), penicillins (081216), tetracyclines (081224), miscellaneous antibiotics (081228), antibiotic composites (081299), antibacterials for eye/ear/nose/throat (520404), and antibacterials for skin/mucous membrane (840404); (2) procedure code: periodontal emergency treatment (91001), subgingival curettage $(91006,91007,91008)$, periodontal flap operation $(91009,91010)$, gingivectomy (91011, 91012, 91013), comprehensive periodontal treatment $(91015,91016)$; and (3) procedure code: scaling $(91003,91004)$ over three times a year. We tracked these exposures and comorbidities till the index date of SLE diagnosis.

\section{Statistical Analysis}

We measured the balance of baseline characteristics in the agematched, sex-matched, and propensity score-matched populations by the absolute standardized difference (ASD), whose value if $<0.1$ was considered a small difference. Student's $t$-test was used to compare variables between cases and controls

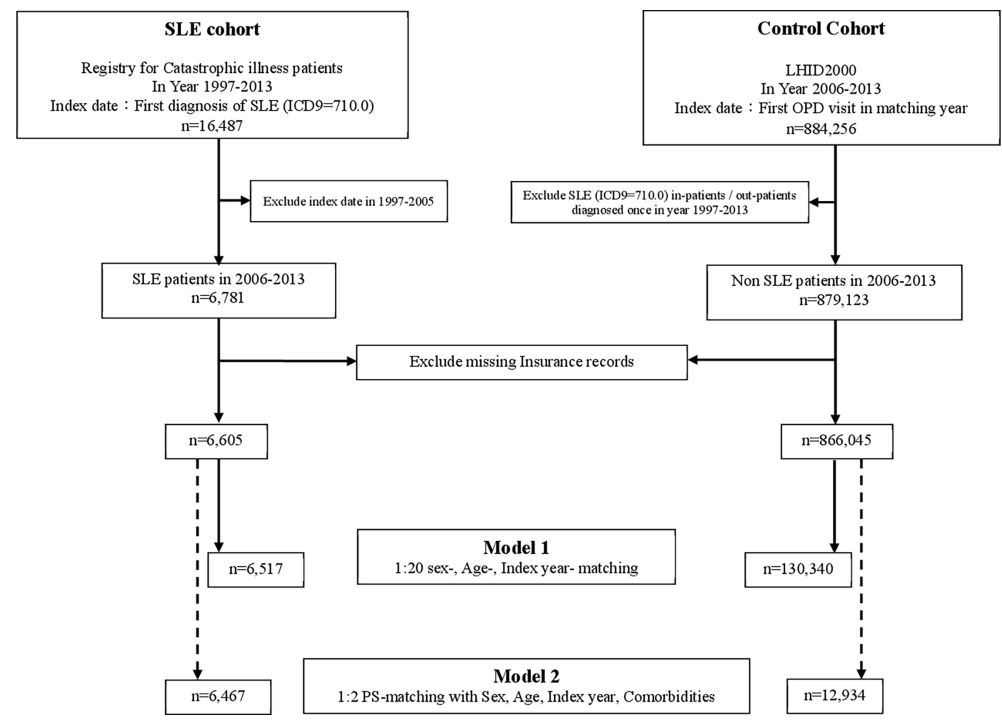

FIGURE 1 | Flowchart. RFCIP, Registry for Catastrophic illness patients; LHID, Longitudinal Health Insurance Research Database; SLE, Systemic lupus erythematosus; OPD, outpatient department; PS-matching, Propensity score matching. 
for continuous variables, and chi-square test was used for comparing categorical variables. We used multivariable conditional logistic regression models to estimate the association between NTS and SLE. To further control for potential confounding factors, all models were adjusted for comorbidities and medications that might be associated with SLE. We used the following four models to estimate the effect of NTS on the risk of SLE: (1) model 1, NTS exposure alone, (2) model 2, NTS exposure + demographic variables, (3) model 3, NTS exposure + demographic variables + medical utilization and comorbidities at baseline in age-matched and sex-matched populations, (4) model 4, a conditional logistic model with NTS exposure alone in propensity score-matched population. Furthermore, we performed subgroup analyses by sex, age, urbanization, insurance amount, cumulative cost of NTS-related visits, and number of NTS visits. To improve the reliability of NTS ICD coding, we enrolled only patients using antibiotics at admission for further ascertainment. Conditional logistic regression of adjusted odds ratios (aORs) with 95\% confidence intervals (CIs) was conducted. All data analyses were performed using SAS ${ }^{\circledR}$ (version 9.4; SAS Institute, Inc., Cary, NC, USA). Statistical significance level was set at $\mathrm{P}$-value $<0.05$ in the two-tailed test.

\section{RESULTS}

Table 1 demonstrates the baseline characteristics of 136,857 and 19,401 patients in 1:20 age-sex matching and in 1:2 PSM analyses, respectively. The mean age of patients in both SLE and non-SLE groups was 37.8 years. There was an obvious female

TABLE 1 | Baseline characteristics among SLE group and non- SLE group.

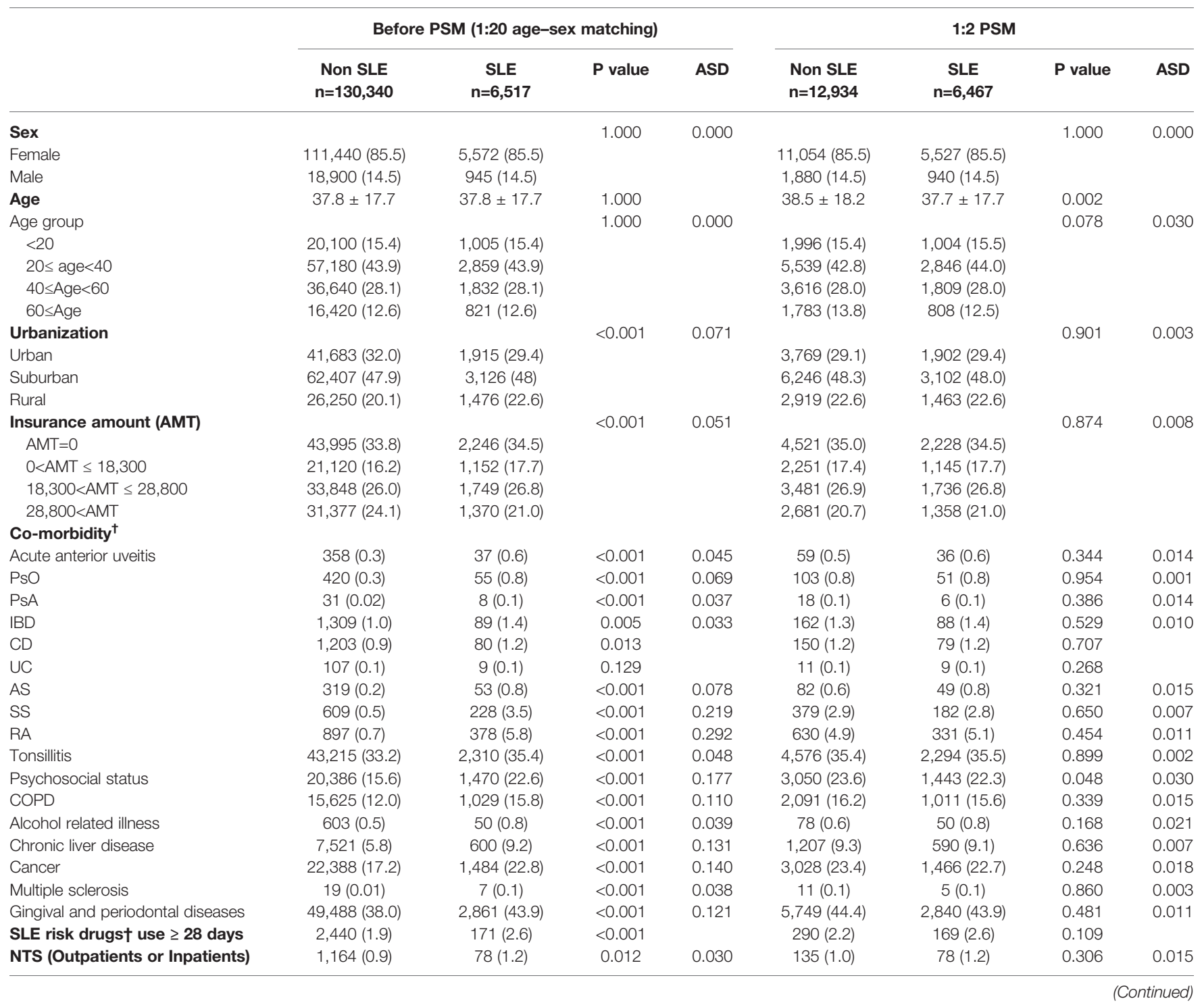


TABLE 1 | Continued

\begin{tabular}{|c|c|c|c|c|c|c|c|c|}
\hline & \multicolumn{4}{|c|}{ Before PSM (1:20 age-sex matching) } & \multicolumn{4}{|c|}{ 1:2 PSM } \\
\hline & $\begin{array}{c}\text { Non SLE } \\
n=130,340\end{array}$ & $\begin{array}{c}\text { SLE } \\
\mathrm{n}=6,517\end{array}$ & $P$ value & ASD & $\begin{array}{l}\text { Non SLE } \\
n=12,934\end{array}$ & $\begin{array}{c}\text { SLE } \\
n=6,467\end{array}$ & P value & ASD \\
\hline Number of NTS visits & & & 0.027 & & & & 0.475 & \\
\hline \multicolumn{9}{|l|}{ (Outpatients or Inpatients) } \\
\hline Non NTS & $129,176(99.1)$ & 6,439 (98.8) & & & 12,799 (99.0) & 6,389 (98.8) & & \\
\hline 1 visit & $724(0.6)$ & $45(0.7)$ & & & $84(0.6)$ & $45(0.7)$ & & \\
\hline$>1$ visit & $440(0.3)$ & $33(0.5)$ & & & $51(0.4)$ & $33(0.5)$ & & \\
\hline $\begin{array}{l}\text { Cumulative cost of NTS-related } \\
\text { visits (New Taiwan dollars) }\end{array}$ & & & $<0.001$ & & & & 0.026 & \\
\hline non NTS & $129,176(99.1)$ & 6,439 (98.8) & & & 12,799 (99.0) & 6,389 (98.8) & & \\
\hline dollars $\leq 333$ & $298(0.2)$ & $14(0.2)$ & & & $36(0.3)$ & $14(0.2)$ & & \\
\hline $333<$ dollars $\leq 425$ & $322(0.2)$ & $13(0.2)$ & & & $38(0.3)$ & $13(0.2)$ & & \\
\hline $425<$ dollars $\leq 1064$ & $267(0.2)$ & $18(0.3)$ & & & $28(0.2)$ & $18(0.3)$ & & \\
\hline $1064<$ dollars & $277(0.2)$ & $33(0.5)$ & & & $33(0.3)$ & $33(0.5)$ & & \\
\hline $\begin{array}{l}\text { Interval between the first NTS } \\
\text { visit and the index date }\end{array}$ & & & 0.016 & & & & 0.186 & \\
\hline non NTS & $129,176(99.1)$ & 6,439 (98.8) & & & 12,799 (99.0) & 6,389 (98.8) & & \\
\hline years $\leq 3.53$ & $289(0.2)$ & $22(0.3)$ & & & $31(0.2)$ & $22(0.3)$ & & \\
\hline $3.53<$ years $\leq 6.08$ & $286(0.2)$ & $24(0.4)$ & & & $32(0.2)$ & $24(0.4)$ & & \\
\hline $6.08<$ years $\leq 8.62$ & $298(0.2)$ & $12(0.2)$ & & & $38(0.3)$ & $12(0.2)$ & & \\
\hline $8.62<$ years & $291(0.2)$ & $20(0.3)$ & & & $34(0.3)$ & $20(0.3)$ & & \\
\hline $\begin{array}{l}\text { Number of NTS visits, } 3 \text { months } \\
\text { interval (Outpatients or } \\
\text { Inpatients) }\end{array}$ & & & 0.022 & & & & 0.589 & \\
\hline Non NTS & $129,176(99.1)$ & 6,439 (98.8) & & & $12,799(99)$ & 6,389 (98.8) & & \\
\hline 1 visit & $835(0.6)$ & $60(0.9)$ & & & $103(0.8)$ & $60(0.9)$ & & \\
\hline$>1$ visit & $329(0.3)$ & $18(0.3)$ & & & $32(0.2)$ & $18(0.3)$ & & \\
\hline \multicolumn{9}{|l|}{$\begin{array}{l}\text { Number of NTS visits, } 3 \text { months } \\
\text { interval (Inpatients only) }\end{array}$} \\
\hline Non NTS & $130,255(99.9)$ & $6,497(99.7)$ & $<0.001$ & & $12,919(99.9)$ & $6,447(99.7)$ & 0.003 & \\
\hline 1 visit & $84(0.1)$ & $20(0.3)$ & & & $15(0.1)$ & $20(0.3)$ & & \\
\hline$>1$ visit & $1(0.001)$ & $0(0.0)$ & & & $0(0.0)$ & $0(0.0)$ & & \\
\hline$\geq 1$ visit & $85(0.1)$ & $20(0.3)$ & & & $15(0.1)$ & $20(0.3)$ & & \\
\hline Non NTS & $130,255()$ & $6,497()$ & & & $12,919()$ & $6,447()$ & & \\
\hline$\geq 1$ visit & 85[] & 20[] & & & 15[] & 20[] & & \\
\hline No antibiotics use during visit & $25(0.02)[29.4]$ & $3(0.05)[15.0]$ & $\begin{array}{l}(<0.001) \\
{[0.147]}\end{array}$ & & $6(0.05)[40.0]$ & $3(0.05)[15.0]$ & $\begin{array}{l}(0.006) \\
{[0.175]}\end{array}$ & \\
\hline IV antibiotics use during visit & $30(0.02)$ [35.3] & $5(0.1)[25.0]$ & & & $5(0.04)$ [33.3] & $5(0.1)[25.0]$ & & \\
\hline Oral antibiotics use during visit & $6(0.005)[7.1]$ & $1(0.02)[5.0]$ & & & $1(0.01)[6.7]$ & $1(0.02)[5.0]$ & & \\
\hline IV + Oral antibiotics use during visit & $24(0.02)$ [28.2] & $11(0.2)[55.0]$ & & & $3(0.02)$ [20.2] & $11(0.2)[55.0]$ & & \\
\hline No antibiotics use $\geq 3$ days during & $26(0.02)[30.6]$ & $4(0.1)[20.0]$ & $(<0.001)$ & & $6(0.05)[40.0]$ & $4(0.1)[20.0]$ & $(0.008)$ & \\
\hline visit & & & {$[0.151]$} & & & & [0.216] & \\
\hline $\begin{array}{l}\text { IV antibiotics use } \geq 3 \text { days during } \\
\text { visit }\end{array}$ & $30(0.02)[35.3]$ & $4(0.1)[20.0]$ & & & $5(0.04)[33.3]$ & $4(0.1)[20.0]$ & & \\
\hline $\begin{array}{l}\text { Oral antibiotics use } \geq 3 \text { days } \\
\text { during visit }\end{array}$ & $5(0.004)[5.9]$ & $1(0.02)[5.0]$ & & & $1(0.01)[6.7]$ & $1(0.02)[5.0]$ & & \\
\hline $\begin{array}{l}\text { IV + Oral antibiotics use } \geq 3 \text { days } \\
\text { during visit }\end{array}$ & $24(0.02)[28.2]$ & $11(0.2)[55.0]$ & & & $3(0.02)[20.0]$ & $11(0.2)[55.0]$ & & \\
\hline $\begin{array}{l}\text { Number of NTS visits, } 3 \text { months } \\
\text { interval (Inpatients only with } \\
\text { antibiotics use) }{ }^{\dagger}\end{array}$ & & & $<0.001$ & & & & $<0.001$ & \\
\hline Non NTS & $130,280(99.95)$ & $6,500(99.7)$ & & & 12,925 (99.9) & $6,450(99.7)$ & & \\
\hline 1 visit & $59(0.05)$ & $17(0.3)$ & & & $9(0.1)$ & $17(0.3)$ & & \\
\hline$>1$ visit & $1(0.001)$ & $0(0.0)$ & & & $0(0.0)$ & $0(0.0)$ & & \\
\hline $\begin{array}{l}\text { Number of NTS visits, } 3 \text { months } \\
\text { interval (Inpatients only with } \\
\text { intravenous antibiotics use) }{ }^{\dagger}\end{array}$ & & & $<0.001$ & & & & $<0.001$ & \\
\hline Non NTS & $130,286(100)$ & $6,501(99.8)$ & & & $12,926(99.9)$ & $6,451(99.8)$ & & \\
\hline 1 visit & $53(0.04)$ & $16(0.2)$ & & & $8(0.1)$ & $16(0.2)$ & & \\
\hline$>1$ visit & $1(0.001)$ & $0(0.0)$ & & & $0(0.0)$ & $0(0.0)$ & & \\
\hline
\end{tabular}




\begin{tabular}{|c|c|c|c|c|c|c|c|c|}
\hline & \multicolumn{4}{|c|}{ Before PSM (1:20 age-sex matching) } & \multicolumn{4}{|c|}{ 1:2 PSM } \\
\hline & $\begin{array}{c}\text { Non SLE } \\
n=130,340\end{array}$ & $\begin{array}{c}\text { SLE } \\
\mathrm{n}=6,517\end{array}$ & P value & ASD & $\begin{array}{l}\text { Non SLE } \\
n=12,934\end{array}$ & $\begin{array}{c}\text { SLE } \\
n=6,467\end{array}$ & $P$ value & ASD \\
\hline $\begin{array}{l}\text { Number of NTS visits, } 3 \text { months } \\
\text { interval (Inpatients only with } \\
\text { oral antibiotics use) }^{+}\end{array}$ & & & $<0.001$ & & & & $<0.001$ & \\
\hline Non NTS & $130,310(100)$ & 6,505 (99.8) & & & $12,930(100)$ & 6,455 (99.8) & & \\
\hline 1 visit & $30(0.02)$ & $12(0.2)$ & & & $4(0.03)$ & $12(0.2)$ & & \\
\hline$>1$ visit & $1(0.001)$ & $0(0.0)$ & & & $0(0.0)$ & $0(0.0)$ & & \\
\hline $\begin{array}{l}\text { Number of NTS visits, } 3 \text { months } \\
\text { interval (Inpatients only with } \\
\text { antibiotics use } \geq 3 \text { days) }\end{array}$ & & & $<0.001$ & & & & 0.001 & \\
\hline Non NTS & $130,281(100)$ & 6,501 (99.8) & & & 12,925 (99.9) & 6,451 (99.8) & & \\
\hline 1 visit & $58(0.04)$ & $16(0.2)$ & & & $9(0.1)$ & $16(0.2)$ & & \\
\hline$>1$ visit & $1(0.001)$ & $0(0.0)$ & & & $0(0.0)$ & $0(0.0)$ & & \\
\hline $\begin{array}{l}\text { Number of NTS visits, } 3 \text { months } \\
\text { interval (Inpatients only with } \\
\text { intravenous antibiotics use } \geq 3 \\
\text { days) }{ }^{\dagger}\end{array}$ & & & $<0.001$ & & & & 0.001 & \\
\hline Non NTS & $130,286(100)$ & 6,502 (99.8) & & & $12,926(99.9)$ & 6,452 (99.8) & & \\
\hline 1 visit & $53(0.04)$ & $15(0.2)$ & & & $8(0.1)$ & $15(0.2)$ & & \\
\hline$>1$ visit & $1(0.001)$ & $0(0.0)$ & & & $0(0.0)$ & $0(0.0)$ & & \\
\hline $\begin{array}{l}\text { Number of NTS visits, } 3 \text { months } \\
\text { interval (Inpatients only with } \\
\text { oral antibiotics use } \geq 3 \text { days) }^{\dagger}\end{array}$ & & & $<0.001$ & & & & $<0.001$ & \\
\hline Non NTS & $130,311(100)$ & 6,505 (99.8) & & & $12,930(100)$ & 6,455 (99.8) & & \\
\hline 1 visit & $29(0.02)$ & $12(0.2)$ & & & $4(0.03)$ & $12(0.2)$ & & \\
\hline$>1$ visit & $0(0.0)$ & $0(0.0)$ & & & $0(0.0)$ & $0(0.0)$ & & \\
\hline
\end{tabular}

${ }^{\dagger}$ Comorbidity and SLE risk drugs (Procainamide, Hydralazine, Isoniazid) were identified from year 2000 to the index date.

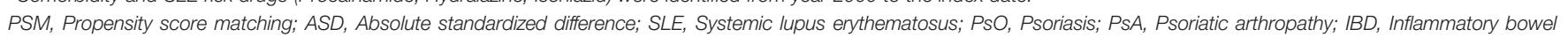
disease; CD, Crohn's disease; UC, Ulcerative colitis; AS, Ankylosing spondylitis; SS, Sjogren's syndrome; RA, Rheumatoid arthritis; COPD, Chronic obstructive pulmonary disease. 口 Antibiotics: 1. Quinolone or 2. Cefixime/Ceftriaxone/Cefotaxime, or 3. Trimethoprime/Sulfamethoxazole, or 4. Ampicillin/Augmentin (Ampicillin +/Clavulanic acid)/Unasyn (Ampicillin + Salbactam).

PSM, Propensity score matching; ASD, Absolute standardized difference; SLE, Systemic lupus erythematosus; NTS, Nontyphoidal Salmonella.

predominance $(85.5 \%)$ in the SLE population, with the majority aged $20-40$ years $(43.9 \%)$, dwelling in suburban regions $(48 \%)$, and having tonsillitis (35.4\%) and gingival and periodontal diseases $(43.9 \%)$. The difference in covariates between both groups was well balanced in PSM analysis with the ASD $<0.1$. The overall ASD revealed a good balance in both SLE and nonSLE groups, whereas it remained mildly uneven in the number of NTS visits subgroup and the interval between the first NTS visit and the index date subgroup with $\mathrm{P}>0.05$ after PSM.

Table 2 shows aORs and 95\% CIs estimated by conditional logistic regression models, with 1:20 age-sex matching and PSM analyses in NTS patients enrolled through ambulatory care or admission. The aOR for SLE was 1.35 (95\% CI=1.07-1.70) in model 1 and 1.35 (95\% CI=1.07-1.71) in model 2. Dwelling in rural $(\mathrm{aOR}=1.20 ; 95 \% \mathrm{CI}=1.12-1.29)$ and suburban $(\mathrm{aOR}=1.08$; 95\% CI=1.02-1.14) areas was associated with a higher risk of SLE in NTS patients than dwelling in urban areas (model 2). NTS patients with rheumatoid arthritis $(\mathrm{aOR}=7.11 ; 95 \% \mathrm{CI}=6.22$ 8.12), multiple sclerosis ( $\mathrm{aOR}=5.44 ; 95 \% \mathrm{CI}=2.15-13.77)$, and Sjogren's syndrome $(\mathrm{aOR}=5.03 ; 95 \% \mathrm{CI}=4.24-5.97)$ had a higher risk of SLE in model 3. The OR in PSM analysis revealed no significant difference between the NTS and control groups in terms of the future incidence of SLE $(\mathrm{aOR}=1.46$; 95\% CI $=0.93$ 2.28 ) in patients who were enrolled through ambulatory care or admission (model 4).
Table 3 demonstrates the subgroup analysis of conditional logistic regression models.

\section{NTS Patients Enrolled Through Ambulatory Care or Admission}

Patients who visited the hospital for NTS more than one time either through ambulatory care or admission had a higher risk of future SLE, with an aOR of $1.51(95 \% \mathrm{CI}=1.06-2.15)$ in model $1,1.52$ (95\% CI=1.06-2.16) in model 2 , and $1.43(95 \%$ $\mathrm{CI}=1.00-2.05)$ in model 3. Furthermore, NTS patients with intensive visits within 3 months were more predisposed to SLE development than patients who had long-term visits, with aOR of 1.44 (95\% CI=1.11-1.88) in model $1,1.45(95 \%$ $\mathrm{CI}=1.11-1.89)$ in model 2 , and 1.35 (95\% $\mathrm{CI}=1.03-1.77)$ in model 3.

A higher cumulative cost of NTS-related visits (>1064 New Taiwan Dollars) was associated with a higher SLE risk, with an aOR of 2.40 (95\% CI=1.67-3.45) in model 1, 2.40 (95\% CI=1.67$3.45)$ in model 2, 2.15 (95\% CI=1.48-3.11) in model 3, and 2.29 (95\% CI=1.30-4.03) in model 4.

Analysis of the interval between the first NTS visit and the index date revealed that an interval of 3.53-6.08 years was associated with a higher SLE risk, with an aOR of $1.68(95 \%$ $\mathrm{CI}=1.11-2.56)$ in model 1 and 1.69 (95\% $\mathrm{CI}=1.11-2.57)$ in model 2. 
TABLE 2 | Unadjusted and adjusted odds ratios for SLE and exposure to NTS.

\begin{tabular}{|c|c|c|c|c|c|}
\hline & \multirow[b]{2}{*}{ Univariable } & \multicolumn{3}{|c|}{ 1:20 age-matched and sex-matched population } & \multirow{2}{*}{$\begin{array}{l}\text { 1:2 PSM population } \\
\text { Model 4: conditional } \\
\text { logistic model with NTS } \\
\text { exposure alone }\end{array}$} \\
\hline & & $\begin{array}{l}\text { Model 1: NTS } \\
\text { exposure alone }\end{array}$ & $\begin{array}{c}\text { Model 2: NTS exposure + } \\
\text { demographic variables }\end{array}$ & $\begin{array}{l}\text { Model 3: model } 2+\text { medical } \\
\text { utilization and comorbidities } \\
\text { at baseline }\end{array}$ & \\
\hline $\begin{array}{l}\text { NTS (Outpatients } \\
\text { or Inpatients) } \\
\text { Urbanization }\end{array}$ & $1.35(1.07-1.70)$ & $1.35(1.07-1.70)$ & $1.35(1.07-1.71)$ & $1.25(0.99-1.59)$ & $1.46(0.93-2.28)$ \\
\hline Urban & Ref. & & Ref. & Ref. & \\
\hline Suburban & 1.09 (1.03-1.16) & & $1.08(1.02-1.14)$ & 1.07 (1.01-1.14) & \\
\hline $\begin{array}{l}\text { Rural } \\
\text { Insurance } \\
\text { amount (AMT) }\end{array}$ & $1.23(1.15-1.32)$ & & $1.20(1.12-1.29)$ & $1.18(1.10-1.27)$ & \\
\hline $\mathrm{AMT}=0$ & Ref. & & Ref. & Ref. & \\
\hline $0<\mathrm{AMT} \leq 18,300$ & $1.04(0.95-1.13)$ & & $1.03(0.95-1.12)$ & $1.03(0.94-1.12)$ & \\
\hline $\begin{array}{l}18,300<\mathrm{AMT} \leq \\
28,800\end{array}$ & $0.97(0.90-1.05)$ & & $0.96(0.89-1.04)$ & $0.95(0.88-1.03)$ & \\
\hline $28,800<$ AMT & $0.81(0.75-0.88)$ & & $0.82(0.76-0.90)$ & $0.82(0.75-0.89)$ & \\
\hline Co-morbidity ${ }^{\dagger}$ & & & & & \\
\hline $\begin{array}{l}\text { Acute anterior } \\
\text { uveitis }\end{array}$ & $2.08(1.48-2.92)$ & & & $1.73(1.21-2.47)$ & \\
\hline $\mathrm{PsO}$ & 2.64 (1.99-3.51) & & & 2.27 (1.67-3.09) & \\
\hline PsA & $5.21(2.39-11.39)$ & & & $1.75(0.71-4.32)$ & \\
\hline IBD & $1.37(1.10-1.70)$ & & & $1.22(0.98-1.52)$ & \\
\hline AS & 3.35 (2.50-4.49) & & & $1.83(1.32-2.54)$ & \\
\hline SS & $8.01(6.84-9.37)$ & & & $5.03(4.24-5.97)$ & \\
\hline $\mathrm{RA}$ & 9.52 (8.38-10.80) & & & $7.11(6.22-8.12)$ & \\
\hline Tonsillitis & $1.12(1.06-1.18)$ & & & $1.02(0.97-1.08)$ & \\
\hline Psychosocial status & 1.65 (1.55-1.76) & & & $1.37(1.28-1.47)$ & \\
\hline COPD & $1.42(1.32-1.52)$ & & & $1.20(1.11-1.29)$ & \\
\hline $\begin{array}{l}\text { Alcohol related } \\
\text { illness }\end{array}$ & $1.67(1.25-2.24)$ & & & $1.16(0.86-1.57)$ & \\
\hline $\begin{array}{l}\text { Chronic liver } \\
\text { disease }\end{array}$ & $1.71(1.56-1.87)$ & & & $1.41(1.28-1.55)$ & \\
\hline Cancer & $1.48(1.39-1.57)$ & & & $1.33(1.25-1.42)$ & \\
\hline Multiple sclerosis & 7.37 (3.10-17.53) & & & $5.44(2.15-13.77)$ & \\
\hline $\begin{array}{l}\text { Gingival and } \\
\text { periodontal } \\
\text { diseases }\end{array}$ & $1.30(1.23-1.37)$ & & & $1.24(1.18-1.31)$ & \\
\hline $\begin{array}{l}\text { SLE risk drugs } \\
\text { use } \geq 28 \text { days }\end{array}$ & $1.45(1.23-1.71)$ & & & $1.30(1.09-1.53)$ & \\
\hline
\end{tabular}

${ }^{+}$Comorbidity and SLE risk drugs (Procainamide, Hydralazine, Isoniazid) were identified from year 2000 to the index date.

PSM, Propensity score matching; SLE, Systemic lupus erythematosus; NTS, Nontyphoidal Salmonella; PsO, Psoriasis; PSA, Psoriatic arthropathy; IBD, Inflammatory bowel disease; CD, Crohn's disease; UC, Ulcerative colitis; AS, Ankylosing spondylitis; SS, Sjogren's syndrome; RA, Rheumatoid arthritis; COPD, Chronic obstructive pulmonary disease.

\section{NTS Patients Enrolled Through Admission Only}

Patients who visited the hospital for NTS at least one time through admission had a higher risk of future SLE, with an aOR of 4.77 (95\% CI=2.92-7.78) in model 1, 4.77 (95\% CI=2.927.79) in model 2, 4.04 (95\% CI=2.44-6.68) in model 3, and 2.88 (95\% CI $=1.43-5.82)$ in model 4 . In the subgroups of antibiotic use, Intravenous (IV) antibiotics use during visit showed significant risk in model 1 (aOR=3.38, 95\% CI= 1.31-8.73), model $2(\mathrm{aOR}=3.33,95 \% \mathrm{CI}=1.29-8.59)$, and model 3 $(\mathrm{aOR}=2.89,95 \% \mathrm{CI}=1.10-7.61)$. In the subgroups of "IV + oral antibiotics use (no matter $\geq 3$ days or not) during visit" showed significant higher risk in 4 models, with aOR ranged from 7.38 to 9.36. Especially in "IV + oral antibiotics use $\geq 3$ days during visit" subgroup (main model), aOR of 9.20 ( $95 \% \mathrm{CI}=4.51$ 18.78), and 7.47 (95\% CI= 2.08-26.82) were observed using $1: 20$ sex-age matching and 1:2 PSM, respectively.
In addition, NTS patients who were admitted with antibiotics use had a higher risk of SLE, with an aOR of 5.71 (95\% CI=3.339.80) in model 1; 5.71 (95\% CI=3.32-9.80) in model 2; 5.00 (95\% $\mathrm{CI}=2.88-8.70)$ in model 3; 3.78 (95\% CI=1.68-8.48) in model 4 .

Finally, NTS patients who were admitted with antibiotics use for at least 3 days had a higher risk of SLE, with an aOR of 5.46 (95\% CI $=3.14-9.50)$ in model $1,5.46$ (95\% CI=3.14-9.50) in model 2, 4.74 (95\% CI=2.69-8.36) in model 3, and 3.56 (95\% $\mathrm{CI}=1.57-8.05)$ in model 4.

\section{DISCUSSION}

In this nationwide, population-based case-control study, we found that the risk of new-onset SLE was 9.20-fold and 7.47fold higher in patients with a history of NTS than in matched controls by 1:20 sex-age matching and PSM analyses, 
TABLE 3 | Unadjusted and adjusted odds ratios for SLE and exposure to NTS.

\begin{tabular}{|c|c|c|c|c|c|}
\hline & \multirow[b]{2}{*}{ Univariable } & \multicolumn{3}{|c|}{ 1:20 age-matched and sex-matched population } & \multirow{2}{*}{$\begin{array}{l}\text { 1:2 PSM population } \\
\text { Model 4: conditional } \\
\text { logistic model with NTS } \\
\text { exposure alone }\end{array}$} \\
\hline & & $\begin{array}{l}\text { Model 1: NTS } \\
\text { exposure alone }\end{array}$ & $\begin{array}{c}\text { Model 2: NTS exposure + } \\
\text { demographic variables }\end{array}$ & $\begin{array}{c}\text { Model 3: model } 2+ \\
\text { medical utilization and } \\
\text { comorbidities at baseline }\end{array}$ & \\
\hline \multicolumn{6}{|c|}{ Number of NTS visits (Outpatients or Inpatients) } \\
\hline non NTS & Ref. & Ref. & Ref. & Ref. & Ref. \\
\hline 1 visit & $1.25(0.92-1.69)$ & $1.25(0.92-1.69)$ & $1.26(0.93-1.70)$ & $1.15(0.84-1.56)$ & $1.35(0.81-2.24)$ \\
\hline$>1$ visit & $1.99(1.24-3.19)$ & $1.51(1.06-2.15)$ & $1.52(1.06-2.16)$ & $1.43(1.00-2.05)$ & $1.63(0.93-2.86)$ \\
\hline \multicolumn{6}{|c|}{ Cumulative cost of NTS-related visits (New Taiwan dollars) (Outpatients or Inpatients) } \\
\hline non NTS & Ref. & Ref. & Ref. & Ref. & Ref. \\
\hline dollars $\leq 333$ & $0.94(0.55-1.61)$ & $0.94(0.55-1.61)$ & $0.95(0.56-1.63)$ & $0.86(0.50-1.50)$ & $0.89(0.43-1.83)$ \\
\hline $333<$ dollars $\leq 425$ & $0.81(0.47-1.41)$ & $0.81(0.47-1.41)$ & $0.81(0.47-1.42)$ & $0.74(0.42-1.30)$ & $0.81(0.38-1.71)$ \\
\hline $425<$ dollars $\leq 1064$ & $1.36(0.84-2.19)$ & $1.36(0.84-2.19)$ & $1.37(0.85-2.21)$ & $1.36(0.84-2.20)$ & $1.59(0.79-3.23)$ \\
\hline $1064<$ dollars & $2.40(1.67-3.45)$ & $2.40(1.67-3.45)$ & $2.40(1.67-3.45)$ & $2.15(1.48-3.11)$ & $2.29(1.30-4.03)$ \\
\hline \multicolumn{6}{|c|}{ Interval between the first NTS visit and the index date (Outpatients or Inpatients) } \\
\hline non NTS & Ref. & Ref. & Ref. & Ref. & Ref. \\
\hline years $\leq 3.53$ & 1.53 (0.99-2.36) & $1.53(0.99-2.36)$ & $1.52(0.99-2.35)$ & $1.46(0.94-2.26)$ & $1.64(0.85-3.17)$ \\
\hline $3.53<$ years $\leq 6.08$ & $1.68(1.11-2.56)$ & $1.68(1.11-2.56)$ & $1.69(1.11-2.57)$ & $1.51(0.99-2.32)$ & 1.79 (0.94-3.39) \\
\hline $6.08<$ years $\leq 8.62$ & $0.81(0.45-1.44)$ & $0.81(0.45-1.44)$ & $0.82(0.46-1.46)$ & $0.75(0.42-1.36)$ & $0.75(0.34-1.68)$ \\
\hline $8.62<$ years & $1.38(0.88-2.18)$ & $1.38(0.88-2.18)$ & $1.40(0.88-2.20)$ & $1.30(0.82-2.06)$ & $1.44(0.72-2.88)$ \\
\hline \multicolumn{6}{|c|}{ Number of NTS visits (3 months interval, Outpatients or Inpatients) } \\
\hline non NTS & Ref. & Ref. & Ref. & Ref. & Ref. \\
\hline Visits $=1$ & $1.44(1.11-1.88)$ & $1.44(1.11-1.88)$ & $1.45(1.11-1.89)$ & $1.35(1.03-1.77)$ & $1.48(0.91-2.40)$ \\
\hline Visits $\geq 2$ & $1.10(0.68-1.77)$ & $1.10(0.68-1.77)$ & $1.11(0.69-1.79)$ & $1.02(0.63-1.65)$ & $1.39(0.70-2.78)$ \\
\hline \multicolumn{6}{|c|}{ Number of NTS visits $\geq 1$ ( 3 months interval, Inpatients only) } \\
\hline & $4.77(2.92-7.78)$ & $4.77(2.92-7.78)$ & $4.77(2.92-7.79)$ & $4.04(2.44-6.68)$ & $2.88(1.43-5.82)$ \\
\hline Non NTS & Ref & Ref & Ref & Ref & Ref \\
\hline $\begin{array}{l}\text { No antibiotics use during } \\
\text { visit }{ }^{+}\end{array}$ & $2.43(0.73-8.07)$ & $2.43(0.73-8.07)$ & $2.44(0.78-8.12)$ & $1.84(0.53-6.32)$ & $1.18(0.29-4.83)$ \\
\hline $\begin{array}{l}\text { IV antibiotics use during } \\
\text { visit }^{+}\end{array}$ & $3.38(1.31-8.73)$ & $3.38(1.31-8.73)$ & 3.33 (1.29-8.59) & $2.89(1.10-7.61)$ & $2.02(0.58-6.99)$ \\
\hline $\begin{array}{l}\text { Oral antibiotics use during } \\
\text { visit }{ }^{+}\end{array}$ & $3.34(0.40-27.77)$ & $3.34(0.40-27.77)$ & $3.34(0.40-27.77)$ & $2.80(0.33-23.70)$ & $2.00(0.13-31.98)$ \\
\hline $\begin{array}{l}\text { IV + Oral antibiotics use } \\
\text { during visit }{ }^{\dagger}\end{array}$ & $9.21(4.51-18.79)$ & $9.21(4.51-18.79)$ & $9.36(4.58-19.11)$ & $8.36(4.01-17.43)$ & 7.38 (2.06-26.49) \\
\hline $\begin{array}{l}\text { No antibiotics use } \geq 3 \\
\text { days during visit }^{\dagger}\end{array}$ & $3.13(1.09-9.00)$ & $3.13(1.09-9.00)$ & $3.13(1.09-9.02)$ & $2.45(0.82-7.30)$ & $1.54(0.42-5.58)$ \\
\hline $\begin{array}{l}\text { IV antibiotics use } \geq 3 \text { days } \\
\text { during visit }{ }^{+}\end{array}$ & $2.70(0.95-7.68)$ & $2.70(0.95-7.68)$ & $2.66(0.94-7.57)$ & $2.28(0.79-6.61)$ & $1.64(0.44-6.14)$ \\
\hline $\begin{array}{l}\text { Oral antibiotics use } \geq 3 \\
\text { days during visit }^{\dagger}\end{array}$ & $4.00(0.47-34.24)$ & $4.00(0.47-34.24)$ & $3.99(0.47-34.16)$ & $3.19(0.36-27.91)$ & $2.00(0.13-31.98)$ \\
\hline $\begin{array}{l}\text { IV + Oral antibiotics use } \geq \\
3 \text { days during visit }{ }^{\dagger *}\end{array}$ & $9.20(4.51-18.78)$ & $9.20(4.51-18.78)$ & 9.35 (4.58-19.10) & $8.35(4.00-17.42)$ & 7.47 (2.08-26.82) \\
\hline \multicolumn{6}{|c|}{ Number of NTS visits $\geq 1$ ( 3 months interval, Inpatients only with antibiotics use) ${ }^{\dagger}$} \\
\hline & $5.71(3.33-9.80)$ & $5.71(3.33-9.80)$ & $5.71(3.32-9.80)$ & $5.00(2.88-8.70)$ & $3.78(1.68-8.48)$ \\
\hline \multicolumn{6}{|c|}{ Number of NTS visits $\geq 1$ ( 3 months interval, Inpatients only with antibiotics use $\geq 3$ days) ${ }^{\dagger}$} \\
\hline & $5.46(3.14-9.50)$ & $5.46(3.14-9.50)$ & $5.46(3.14-9.50)$ & $4.74(2.69-8.36)$ & $3.56(1.57-8.05)$ \\
\hline
\end{tabular}

*Main model.

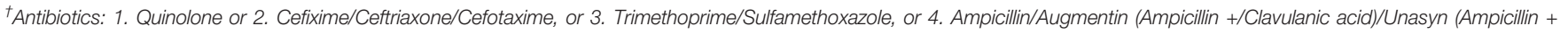
Salbactam).

PSM, Propensity score matching; SLE, Systemic lupus erythematosus; NTS, Nontyphoidal Salmonella.

respectively. Consistent with epidemiological observations, Comorbidities such as Sjogren's syndrome (24) (ASD = 0.219), Rheumatoid arthritis (25) (ASD = 0.292), Psychosocial status (26) $(\mathrm{ASD}=0.177)$, gingival and periodontal disease $(27)(\mathrm{ASD}=$ 0.121 ) etc. were significantly more predominant in SLE patients than in general population (Table $\mathbf{1}$ ).

Despite different models rendered different odds ratio in Table 2, our data implied that SLE was prone to rise among NTS-infected patients who had Sjogren's syndrome, Rheumatoid arthritis,
Multiple sclerosis, and who lived in rural area rather than suburban and urban area. In Table 3, among ambulatory care or admission patients, the more intensive NTS visit in one NTS infection episode, the higher of NTS cumulative cost gave rise to higher odds ratio of future SLE, which largely occurred among 3 to 6 years after NTS infection. Among admission only patients, the more critical NTS infection is (who needed hospitalization and with both IV and oral antibiotics use), the higher odds of future SLE will be. Despite study illustrated that Salmonellosis of long duration was 
characterized by hyperactivity of autoimmune reactions (28), there was no advanced description on the duration from severe Salmonellosis to the onset of autoimmune disease.

Environmental exposures other than infections might also depend on the level of urbanization. First, the smoking behavior of Taiwanese individuals was estimated to be $71.63 \%$ and was largely distributed (47.94\%) in suburban area (29), which correlated with our observation. Second, dwelling in areas in which the content of particulate matter $(2.5 \mu \mathrm{m})$ was over $35 \mu \mathrm{g} /$ $\mathrm{m}^{3}$ was associated with $15 \%$ higher long-term mortality risk according to the air quality guideline (30); such areas included many suburban and rural areas in Taiwan in 2013 (31) and are linked to many autoimmune diseases (7). Third, heavy metals were largely spatially distributed in the soil of rural townships during 1982-1986 in Taiwan (32). Major pollution events (33) in the past, such as aluminum slag, electric arc furnace dust pollution, and rice cadmium toxicity, largely in rural areas possibly trigger the onset of SLE or aggravate the condition. Last, low-income households that can afford only low insurance amounts are mainly located in rural areas (34), and they accounted for the higher prevalence of SLE in this study.

Other autoimmune diseases, such as Sjogren's syndrome, Rheumatoid arthritis, Multiple sclerosis, were prone to develop high risk of SLE in NTS infected patients in our study. Changes in symbiosis and dysbiosis of microbiome could flare autoimmune diseases (35). In patients with Sjogren's syndrome, the changes of oral microbiome in glandular and mucosal tissues brought about activated plasma cell and CD4+ cells that ruined the secretory acini of salivary glands. Similarly, increased levels of IFN- $\gamma$ and IL-17 caused by infiltration of CD4+ cells and dendritic cells in the conjunctiva causing ocular disease (36). On the other hand, in patients with Rheumatoid arthritis, dental, saliva, or gut microbiome was distinguished from healthy controls (37). Its HLA-DRB1 molecules, trans-membrane glycoproteins of macrophages and dendritic cells that present antigens to $\mathrm{T}$ cells, appear to forge the microbiome signature and build an inflammatory environment in animal study (38). Furthermore, gut microbiome shown to be altered in patients with relapsing Multiple sclerosis, causing changes of the metabolic pathways or the Gut-associated lymphoid tissues (GALTs) and aggravation of inflammatory demyelination in central nervous system (39).

Among environmental factors in SLE pathogenesis, microbiome has gained much attention in recent years. The mechanism by which NTS infection increases the risk of SLE remains unclear and disputed. Yet, we propose that long-term NTS stimulates abundant proinflammatory cytokines and high oxidative stress through innate and adaptive immune response, leading to the onset of SLE.

\section{Interaction Between Innate and Adaptive Immune Systems}

After reaching the lower intestine, salmonella bacteria translocate across Peyer's patches where they are engulfed by phagocytic cells. Salmonella are recognized by patternrecognition receptors, triggering cascades toward the induction of interleukin (IL)-1 $\beta$, IL-18, and IL-23; cell pyroptosis and inefficient clearance of phagocytized bacteria (40). Infections by bacteria such as Escherichia coli and Salmonella are wellknown triggers of pyroptosis (41), which may play a pathogenic role in releasing host nuclear autoantigens in SLE (18).

\section{Type I Interferon (IFN-I)}

The autoantigens generated from uncleared apoptotic cells and neutrophil extracellular traps may induce the proliferation and differentiation of autoreactive B cells to produce autoantibodies, which then participate in the formation of immune complexes and tissue injuries. Both autoantigens and immune complexes promote the production of IFN-I by activating plasmacytoid dendritic cell signaling via amyloid/DNA complexes $(42,43)$. IFN-I hampers the clearance of apoptotic cells by macrophages and induces B cells to produce autoantibodies (44). In addition, the immune complexes containing antinuclear autoantibodies could provoke plasmacytoid dendritic cells to produce more IFN-I, leading to SLE $(45,46)$. Therefore, IFN-I has been recognized as the central pathogenic cytokine in SLE onset (47).

\section{IL-17, IL-18, and IFN- $\gamma$}

During NTS infection, there is a massive release of proinflammatory cytokines including IL-23 and IL-18 (40). Antigen-presenting cells produce IL-23, which induces and maintains the activation of Th17 cells (48), and induces various innate and adaptive immune response cells, including innate lymphoid cells (49) and Th17 cells (50) to release the proinflammatory cytokines IL-17 and IL-22. IL-17, in conjunction with $\mathrm{B}$ cell activating factor, promotes the activation and proliferation of $\mathrm{B}$ cells along with their antibody production and class switching $(51,52)$. Lupus patients have been reported to have increased serum levels of IL-17 (53) and IL-17-producing cells in the peripheral blood $(54,55)$. IL-18 is vital in stimulating Th1 cells to release IFN- $\gamma$ (56), which plays a critical role in macrophage activation for the control of persistent salmonella infection (57-59). At the same time, the elevation of IL-17 and IL18 in SLE patients may trigger the inflammatory process, and an increase in the IL-18/IL-4 ratio and mRNA levels of IFN- $\gamma / \mathrm{IL}-4$ (60) tilts the cytokine profile toward inflammatory cascades (53).

In real world practice, we prescribed intravenous antibiotics to patients with severe NTS infection and transited to oral antibiotics when discharged in Taiwan $(61,62)$. The optimal antibiotic treatment duration for severe NTS gastrointestinal infection was 3-7 days and for NTS bacteremia ranges from 2 weeks (in healthy individuals) to 6 weeks (in immunocompromised patients) (63). Therefore, the data in "IV + Oral antibiotics use $\geq 3$ days during visit" group (main model) was more persuasive than that in other groups. Despite low sample size gave rise to low statistical power, the high aOR ( $\mathrm{aOR}=7.47,95 \% \mathrm{CI}=2.08-26.82)$ in "IV + Oral antibiotics use $\geq 3$ days during visit" subgroup using PSM strongly implied a correlation between NTS infection and future SLE development.

The advantages of using NHIRD in research include the availability of a large sample size, population-based data, and long-term comprehensive follow-up data. Our study is representative of the general population, and the possible measurable confounding factors of SLE were adjusted through matching with age, sex, demographic variables, and comorbidities 
through PSM. In addition, we strengthen the validity of the coding of NTS-associated diseases by filtering the NTS patients into those for whom only admission records data were available and those who were administered antibiotics to treat NTS infection. Therefore, the diagnosis of symptomatic NTS infection in our study population can be considered to be reliable.

Several limitations should be considered when interpreting our findings. First, epidemiologic evaluation of evident NTS infection was challenging as many infections were not clinically recognized. The use of retrospective ICD-9-based methods to select study groups of NTS infection might have led to selection bias. Second, NHIRD lacked information on smoking status, a well-known confounder of SLE and the most common risk factor for COPD development (64). Therefore, we managed to apply COPD prevalence as a proxy for smoking status (29). Third, non-differential misclassification bias on the diagnosis of NTS, a dichotomous variable, may exist. However, such bias always drives the result toward the null, and attenuates real effect estimates (65). Fourth, this was a single-country evaluation. Thus, our findings may not be applicable to non-Asian ethnic groups. Finally, the relation between symptomatic NTS and SLE was only demonstrated based on epidemiological evidence.

\section{CONCLUSION}

Exposure to NTS infection may be associated with an increased risk SLE and the risk increased with the individual severity of NTS infection. More epidemiologic and in vivo investigations are

\section{REFERENCES}

1. Carter EE, Barr SG, Clarke AE. The Global Burden of SLE: Prevalence, Health Disparities and Socioeconomic Impact. Nat Rev Rheumatol (2016) 12 (10):605-20. doi: 10.1038/nrrheum.2016.137

2. Yen EY, Singh RR. Brief Report: Lupus-An Unrecognized Leading Cause of Death in Young Females: A Population-Based Study Using Nationwide Death Certificates, 2000-2015. Arthritis Rheumatol (Hoboken NJ) (2018) 70 (8):1251-5. doi: 10.1002/art.40512

3. Shah D, Mahajan N, Sah S, Nath SK, Paudyal B. Oxidative Stress and its Biomarkers in Systemic Lupus Erythematosus. J Biomed Sci (2014) 21(1):23. doi: 10.1186/1423-0127-21-23

4. Deapen D, Escalante A, Weinrib L, Horwitz D, Bachman B, Roy-Burman P, et al. A Revised Estimate of Twin Concordance in Systemic Lupus Erythematosus. Arthritis Rheum (1992) 35(3):311-8. doi: 10.1002/art.1780350310

5. Cooper GS, Miller FW, Pandey JP. The Role of Genetic Factors in Autoimmune Disease: Implications for Environmental Research. Environ Health Perspect (1999) 107 Suppl 5(Suppl 5):693-700. doi: 10.1289/ ehp.99107s5693

6. Kuo CF, Grainge MJ, Valdes AM, See LC, Luo SF, Yu KH, et al. Familial Aggregation of Systemic Lupus Erythematosus and Coaggregation of Autoimmune Diseases in Affected Families. JAMA Internal Med (2015) 175 (9):1518-26. doi: 10.1001/jamainternmed.2015.3528

7. Gulati G, Brunner HI. Environmental Triggers in Systemic Lupus Erythematosus. Semin Arthritis Rheum (2018) 47(5):710-7. doi: 10.1016/ j.semarthrit.2017.10.001

8. Sun F, Chen Y, Wu W, Guo L, Xu W, Chen J, et al. Varicella Zoster Virus Infections Increase the Risk of Disease Flares in Patients With SLE: A Matched Cohort Study. Lupus Sci Med (2019) 6(1):e000339. doi: 10.1136/lupus-2019-000339 warranted to examine whether causality present between NTS infection and its trigger on SLE.

\section{DATA AVAILABILITY STATEMENT}

The original contributions presented in the study are included in the article/supplementary material. Further inquiries can be directed to the corresponding authors.

\section{ETHICS STATEMENT}

The Institutional Review Board of Taichung Veterans General Hospital (TCVGH CE14149B-1) approved this study. Written informed consent for participation was not required for this study in accordance with the national legislation and the institutional requirements.

\section{AUTHOR CONTRIBUTIONS}

Study conception and design: Y-MH, RC, H-HC, JC-CW. Acquisition of data: H-HC. Analysis and interpretation of data: T-YT, C-YY, Y-MH, RC, H-HC, JC-CW. Writing (original draft preparation): T-YY, C-YY. Writing (review and editing): T-YT, C-YY, H-HC, Y-MH, RC, JC-CW. All authors contributed to the article and approved the submitted version.
9. Navarra SV, Leynes MS. Infections in Systemic Lupus Erythematosus. Lupus (2010) 19(12):1419-24. doi: 10.1177/0961203310374486

10. Azzouz D, Omarbekova A, Heguy A, Schwudke D, Gisch N, Rovin BH, et al. Lupus Nephritis is Linked to Disease-Activity Associated Expansions and Immunity to a Gut Commensal. Ann Rheumat Dis (2019) 78(7):947-56. doi: 10.1136/annrheumdis-2018-214856

11. Sela O, El-Roeiy A, Isenberg DA, Kennedy RC, Colaco CB, Pinkhas J, et al. A Common Anti-Dna Idiotype in Sera of Patients With Active Pulmonary Tuberculosis. Arthritis Rheumatol (1987) 30(1):50-6. doi: 10.1002/art.1780300107

12. Balbi GGM, Machado-Ribeiro F, Marques CDL, Signorelli F, Levy RA. The Interplay Between Tuberculosis and Systemic Lupus Erythematosus. Curr Opin Rheumatol (2018) 30(4):395-402. doi: 10.1097/BOR.0000000000000493

13. Soloski MJ, Metcalf ES. Salmonella as an Inducer of Autoimmunity. EcoSal Plus (2007) 2(2). doi: 10.1128/ecosalplus.8.8.13

14. Hannu T, Mattila L, Siitonen A, Leirisalo-Repo M. Reactive Arthritis Following an Outbreak of Salmonella Typhimurium Phage Type 193 Infection. Ann Rheumat Dis (2002) 61(3):264-6. doi: 10.1136/ard.61.3.264

15. Kerstein A, Schüler S, Cabral-Marques O, Fazio J, Häsler R, Müller A, et al. Environmental Factor and Inflammation-Driven Alteration of the Total Peripheral T-Cell Compartment in Granulomatosis With Polyangiitis. J Autoimmun (2017) 78:79-91. doi: 10.1016/j.jaut.2016.12.004

16. Locht H, Mølbak K, Krogfelt KA. High Frequency of Reactive Joint Symptoms After an Outbreak of Salmonella Enteritidis. J Rheumatol (2002) 29(4):767-71.

17. Costa-Reis P, Nativ S, Isgro J, Rodrigues T, Yildirim-Toruner C, Starr A, et al. Major Infections in a Cohort of 120 Patients With Juvenile-Onset Systemic Lupus Erythematosus. Clin Immunol (Orlando Fla) (2013) 149(3):442-9. doi: 10.1016/j.clim.2013.08.009

18. Qiu CC, Caricchio R, Gallucci S. Triggers of Autoimmunity: The Role of Bacterial Infections in the Extracellular Exposure of Lupus Nuclear 
Autoantigens. Front Immunol (2019) 10:2608. doi: 10.3389/fimmu. 2019.02608

19. Gould LH, Walsh KA, Vieira AR, Herman K, Williams IT, Hall AJ, et al. Surveillance for Foodborne Disease Outbreaks - United States, 1998-2008. Morbid Mortal Weekly Rep Surveillance Summaries (Washington DC 2002) (2013) 62(2):1-34.

20. Majowicz SE, Musto J, Scallan E, Angulo FJ, Kirk M, O'Brien SJ, et al. The Global Burden of Nontyphoidal Salmonella Gastroenteritis. Clin Infect Dis An Off Publ Infect Dis Soc America (2010) 50(6):882-9. doi: 10.1086/650733

21. Issac JM, Mohamed YA, Bashir GH, Al-Sbiei A, Conca W, Khan TA, et al. Induction of Hypergammaglobulinemia and Autoantibodies by Salmonella Infection in MyD88-Deficient Mice. Front Immunol (2018) 9:1384. doi: 10.3389/fimmu.2018.01384

22. Bae SC, Lee YH. Causal Association Between Periodontitis and Risk of Rheumatoid Arthritis and Systemic Lupus Erythematosus: A Mendelian Randomization. Z fur Rheumatol (2020) 79(9):929-36. doi: 10.1007/s00393019-00742-w

23. Weinstein A. Drug-Induced Systemic Lupus Erythematosus. Prog Clin Immunol (1980) 4:1-21.

24. Pasoto SG, Adriano de Oliveira Martins V, Bonfa E. Sjögren's Syndrome and Systemic Lupus Erythematosus: Links and Risks. Open Access Rheumatol Res $\operatorname{Rev}(2019)$ 11:33-45. doi: 10.2147/oarrr.S167783

25. Lee YH, Bae SC, Song GG. Meta-Analysis of Associations Between Functional HLA-G Polymorphisms and Susceptibility to Systemic Lupus Erythematosus and Rheumatoid Arthritis. Rheumatol Int (2015) 35(6):953-61. doi: 10.1007/ s00296-014-3155-3

26. Meszaros ZS, Perl A, Faraone SV. Psychiatric Symptoms in Systemic Lupus Erythematosus: A Systematic Review. J Clin Psychiatry (2012) 73(7):9931001. doi: 10.4088/JCP.11r07425

27. Zhong HJ, Xie HX, Luo XM, Zhang EH. Association Between Periodontitis and Systemic Lupus Erythematosus: A Meta-Analysis. Lupus (2020) 29 (10):1189-97. doi: 10.1177/0961203320938447

28. Frolov VM, Iushchuk ND, Peresadin NA. Immunologic Status of Patients With Salmonella Infections. Klinicheskaia Meditsina (1989) 67(10):108-11.

29. Chung W-S, Kung P-T, Chang H-Y, Tsai W-C. Demographics and Medical Disorders Associated With Smoking: A Population-Based Study. BMC Public Health (2020) 20(1):702. doi: 10.1186/s12889-020-08858-4

30. World Health Organization. Regional Office for E. Air Quality Guidelines: Global Update 2005: Particulate Matter, Ozone, Nitrogen Dioxide and Sulfur Dioxide. Copenhagen: World Health Organization. Regional Office for Europe (2006).

31. EP A. Real-Time Air Quality Pollution Indicators. Taipei: Environmental Protection Administration, Executive Yuan, R.O.C. (Taiwan) (2021).

32. Huang HH, Huang JY, Lung CC, Wu CL, Ho CC, Sun YH, et al. Cell-Type Specificity of Lung Cancer Associated With Low-Dose Soil Heavy Metal Contamination in Taiwan: An Ecological Study. BMC Public Health (2013) 13:330. doi: 10.1186/1471-2458-13-330

33. Liu GY, Wang JD, Cao KT. Major Pollution Events in Taiwan. Taipei: CommonWealth Magazine (2020).

34. MoHa W. The Number of Low-Income Households and the Number of People Divided by Township and City. Taipei: Ministry of Health and Welfare, Executive Yuan, R.O.C. (Taiwan) (2021).

35. Nikitakis NG, Papaioannou W, Sakkas LI, Kousvelari E. The AutoimmunityOral Microbiome Connection. Oral Dis (2017) 23(7):828-39. doi: 10.1111/ odi. 12589

36. Stern ME, Schaumburg CS, Pflugfelder SC. Dry Eye as a Mucosal Autoimmune Disease. Int Rev Immunol (2013) 32(1):19-41. doi: 10.3109/ 08830185.2012.748052

37. Zhang X, Zhang D, Jia H, Feng Q, Wang D, Liang D, et al. The Oral and Gut Microbiomes are Perturbed in Rheumatoid Arthritis and Partly Normalized After Treatment. Nat Med (2015) 21(8):895-905. doi: 10.1038/nm.3914

38. Gomez A, Luckey D, Yeoman CJ, Marietta EV, Berg Miller ME, Murray JA, et al. Loss of Sex and Age Driven Differences in the Gut Microbiome Characterize Arthritis-Susceptible 0401 Mice But Not Arthritis-Resistant 0402 Mice. PloS One (2012) 7(4):e36095. doi: 10.1371/journal.pone.0036095

39. Ochoa-Repáraz J, Kirby TO, Kasper LH. The Gut Microbiome and Multiple Sclerosis. Cold Spring Harbor Perspect Med (2018) 8(6). doi: 10.1101/ cshperspect.a029017
40. Behnsen J, Perez-Lopez A, Nuccio SP, Raffatellu M. Exploiting Host Immunity: The Salmonella Paradigm. Trends Immunol (2015) 36(2):11220. doi: $10.1016 /$ j.it.2014.12.003

41. Liu X, Lieberman J. A Mechanistic Understanding of Pyroptosis: The Fiery Death Triggered by Invasive Infection. Adv Immunol (2017) 135:81-117. doi: 10.1016/bs.ai.2017.02.002

42. Sepulveda-Toepfer JA, Pichler J, Fink K, Sevo M, Wildburger S, Mudde-Boer LC, et al. TLR9-Mediated Activation of Dendritic Cells by CD32 Targeting for the Generation of Highly Immunostimulatory Vaccines. Hum Vaccines Immunother (2019) 15(1):179-88. doi: 10.1080/21645515.2018.1514223

43. Gallo PM, Rapsinski GJ, Wilson RP, Oppong GO, Sriram U, Goulian M, et al. Amyloid-DNA Composites of Bacterial Biofilms Stimulate Autoimmunity. Immunity (2015) 42(6):1171-84. doi: 10.1016/j.immuni. 2015.06.002

44. Mauri C, Menon M. The Many Faces of Type I Interferon in Systemic Lupus Erythematosus. J Clin Invest (2015) 125(7):2562-4. doi: 10.1172/JCI82574

45. Li H, Fu YX, Wu Q, Zhou Y, Crossman DK, Yang P, et al. Interferon-Induced Mechanosensing Defects Impede Apoptotic Cell Clearance in Lupus. J Clin Invest (2015) 125(7):2877-90. doi: 10.1172/JCI81059

46. Ma C, Xia Y, Yang Q, Zhao Y. The Contribution of Macrophages to Systemic Lupus Erythematosus. Clin Immunol (Orlando Fla) (2019) 207:1-9. doi: 10.1016/j.clim.2019.06.009

47. Chan VS, Nie YJ, Shen N, Yan S, Mok MY, Lau CS. Distinct Roles of Myeloid and Plasmacytoid Dendritic Cells in Systemic Lupus Erythematosus. Autoimmun Rev (2012) 11(12):890-7. doi: 10.1016/ j.autrev.2012.03.004

48. Langrish CL, Chen Y, Blumenschein WM, Mattson J, Basham B, Sedgwick JD, et al. IL-23 Drives a Pathogenic T Cell Population That Induces Autoimmune Inflammation. J Exp Med (2005) 201(2):233-40. doi: 10.1084/jem.20041257

49. Takatori H, Kanno Y, Watford WT, Tato CM, Weiss G, Ivanov II, et al. Lymphoid Tissue Inducer-Like Cells Are an Innate Source of IL-17 and IL-22. J Exp Med (2009) 206(1):35-41. doi: 10.1084/jem.20072713

50. Ouyang W, Kolls JK, Zheng Y. The Biological Functions of T Helper 17 Cell Effector Cytokines in Inflammation. Immunity (2008) 28(4):454-67. doi: 10.1016/j.immuni.2008.03.004

51. Doreau A, Belot A, Bastid J, Riche B, Trescol-Biemont MC, Ranchin B, et al. Interleukin 17 Acts in Synergy With B Cell-Activating Factor to Influence B Cell Biology and the Pathophysiology of Systemic Lupus Erythematosus. Nat Immunol (2009) 10(7):778-85. doi: 10.1038/ni.1741

52. Mitsdoerffer M, Lee Y, Jäger A, Kim HJ, Korn T, Kolls JK, et al. Proinflammatory T Helper Type 17 Cells are Effective B-Cell Helpers. Proc Natl Acad Sci USA (2010) 107(32):14292-7. doi: 10.1073/pnas.1009234107

53. Wong CK, Ho CY, Li EK, Lam CW. Elevation of Proinflammatory Cytokine (IL-18, IL-17, IL-12) and Th2 Cytokine (IL-4) Concentrations in Patients With Systemic Lupus Erythematosus. Lupus (2000) 9(8):589-93. doi: 10.1191/ 096120300678828703

54. Apostolidis SA, Lieberman LA, Kis-Toth K, Crispín JC, Tsokos GC. The Dysregulation of Cytokine Networks in Systemic Lupus Erythematosus. J Interferon Cytokine Res Off J Int Soc Interferon Cytokine Res (2011) 31(10):769-79. doi: 10.1089/jir.2011.0029

55. Crispín JC, Oukka M, Bayliss G, Cohen RA, Van Beek CA, Stillman IE, et al. Expanded Double Negative T Cells in Patients With Systemic Lupus Erythematosus Produce IL-17 and Infiltrate the Kidneys. J Immunol (Baltimore Md 1950) (2008) 181(12):8761-6. doi: 10.4049/jimmunol. 181.12.8761

56. O'Donnell H, Pham OH, Li LX, Atif SM, Lee SJ, Ravesloot MM, et al. Toll-Like Receptor and Inflammasome Signals Converge to Amplify the Innate Bactericidal Capacity of T Helper 1 Cells. Immunity (2014) 40(2):213-24. doi: 10.1016/j.immuni.2013.12.013

57. Monack DM, Bouley DM, Falkow S. Salmonella Typhimurium Persists Within Macrophages in the Mesenteric Lymph Nodes of Chronically Infected Nramp1+/+ Mice and can be Reactivated by IFNgamma Neutralization. J Exp Med (2004) 199(2):231-41. doi: 10.1084/ jem.20031319

58. Mastroeni P, Clare S, Khan S, Harrison JA, Hormaeche CE, Okamura H, et al Interleukin 18 Contributes to Host Resistance and Gamma Interferon Production in Mice Infected With Virulent Salmonella Typhimurium. Infect Immun (1999) 67(2):478-83. doi: 10.1128/IAI.67.2.478-483.1999 
59. de Jong HK, Parry CM, van der Poll T, Wiersinga WJ. Host-Pathogen Interaction in Invasive Salmonellosis. PloS Pathogens (2012) 8(10): e1002933. doi: 10.1371/journal.ppat.1002933

60. Lit LC-W, Wong C-K, Li EK-M, Tam L-S, Lam CW-K, Lo Y-MD. Elevated Gene Expression of Th1/Th2 Associated Transcription Factors Is Correlated With Disease Activity in Patients With Systemic Lupus Erythematosus. J Rheumatol (2006) 34(1):89-96.

61. Lee CC, Chen PL, Hsieh CC, Yang CY, Lin CH, Ko WC. Is Early Oral Antimicrobial Switch Useful for Less Critically Ill Adults With CommunityOnset Bacteraemia in Emergency Departments? Antibiotics (Basel Switzerland) (2020) 9(11):807. doi: 10.3390/antibiotics9110807

62. Dhanoa A, Fatt QK. Non-Typhoidal Salmonella Bacteraemia: Epidemiology, Clinical Characteristics and Its' Association With Severe Immunosuppression. Ann Clin Microbiol Antimicrob (2009) 8:15. doi: 10.1186/1476-0711-8-15

63. UPTODATE. Nontyphoidal Salmonella: Gastrointestinal Infection and Carriage. Waltham, MA, United States: Wolters Kluwer (2021).

64. Ramsey SD, Hobbs FDR. Chronic Obstructive Pulmonary Disease. Risk Factors Outcome Trials (2006) 3(7):635-40. doi: 10.1513/pats.200603-094SS

65. Burstyn I, Yang Y, Schnatter AR. Effects of Non-Differential Exposure Misclassification on False Conclusions in Hypothesis-Generating Studies, Vol.
11. Basel, Switzerland: Multidisciplinary Digital Publishing Institute (2014). pp. 10951-66.

Conflict of Interest: The authors declare that the research was conducted in the absence of any commercial or financial relationships that could be construed as a potential conflict of interest.

Publisher's Note: All claims expressed in this article are solely those of the authors and do not necessarily represent those of their affiliated organizations, or those of the publisher, the editors and the reviewers. Any product that may be evaluated in this article, or claim that may be made by its manufacturer, is not guaranteed or endorsed by the publisher.

Copyright (C) 2021 Tu, Yeh, Hung, Chang, Chen and Wei. This is an open-access article distributed under the terms of the Creative Commons Attribution License (CC BY). The use, distribution or reproduction in other forums is permitted, provided the original author(s) and the copyright owner(s) are credited and that the original publication in this journal is cited, in accordance with accepted academic practice. No use, distribution or reproduction is permitted which does not comply with these terms. 